\title{
Ganglioside Extraction from Erythrocytes: a Comparison Study
}

\author{
Wen-Qi Wang and Anders Gustafson ${ }^{\dagger}$
}

Department of Cell Biology I, University Hospital, S-221 85 Lund, Sweden

\begin{abstract}
Wang, W.-Q. and Gustafson, A., 1995. Ganglioside Extraction from Erythrocytes: a Comparison Study. - Acta Chem. Scand. 49: 929-936 @ Acta Chemica Scandinavica 1995.

Several methods for ganglioside extraction from erythrocytes have been compared. Our results show that ganglioside extraction is unfavourably affected by the addition of the solvents as a mixture and by the use of less polar solvents and by a lower total solvent-to-sample ratio. In accordance with our previous observation on cholesterol and phospholipids, the distribution of gangliosides could be uneven in an apparently monophasic extraction solvent mixture. The uneven distribution occurred during and also after the extraction (in filtration and centrifugation). In the recommended method using 19 volumes of methanol/chloroform $(2: 1)$ solvent in a one-step extraction, the above disadvantages in ganglioside extraction and quantification are kept under control. This method appears simple and it gives a high recovery of gangliosides.
\end{abstract}

There are a number of methods available for lipid extraction. The procedures are largely variable in their use of total solvent-to-sample ratio and alcohol-to-non-polar solvent ratio, of different alcohols (methanol, ethanol and isopropyl alcohol) and non-polar solvents (chloroform, hexane, cyclohexane), of one-step or repeated extraction procedures, and in the method of solvent addition (as a mixture or separately). It has been found that some methods, such as those of Folch ${ }^{1}$ and Bligh and Dyer, ${ }^{2}$ fail to give complete extraction of cholesterol and phospholipids $^{3-7}$ and that the recovery of these lipids by these two methods is variable. ${ }^{6}$ Even so, these methods remain popular for the extraction not only of cholesterol, phospholipids, but also of polar gangliosides from various tissues.

Recently, we found that in lipid extraction with a methanol-chloroform-water solvent mixture, the solvent and the lipids (cholesterol and phospholipids) could be unevenly distributed between the extract supernatant and the solvent remaining in the residue. ${ }^{8}$ After filtration, an uneven distribution also appeared between the filtrate and filtration medium, as well as after centrifugation between the top and bottom solvent fractions. ${ }^{9}$ We suggested that such uneven distributions could well be the reason for an observed large variation in the cholesterol and phospholipid extractions. ${ }^{8}$

Gangliosides, a group of glycosphingolipids more polar than cholesterol and phospholipids (owing to their sialic acid residues), are widely distributed among tissues. They

\footnotetext{
To whom correspondence should be addressed.
}

participate in the process of signal translocation through membranes and regulate cell differentiation and proliferation (reviewed in Ref. 10). The importance of gangliosides in the pathogenesis of atherosclerosis has recently also attracted attention. ${ }^{11}$ It was therefore of great importance to test how the ganglioside extraction might be affected by the previously discovered uneven distribution during and after a methanol-chloroform-water extraction. The present comparison study became possible after the introduction of our recently developed procedures for ganglioside purification and sialic acid quantification. ${ }^{12}$

\section{Experimental and results}

I. Materials. Analytical reagent-grade chemicals and solvents were used. Organic solvents, Silica Gel 60 (230$400 \mathrm{mesh}$ ), and HPTLC Silica Gel 60 plate were obtained from Merck (Germany). $N$-Acetylneuraminic acid was used as a sialic acid standard and was obtained from Sigma (St. Louis, USA). Ganglioside standards were the kind gift of Pam Fredman (Department of Psychiatry and Neurochemistry, University Hospital of Göteborg, Sweden).

\section{General procedures}

(1) Cell preparation. Blood from fasting donors was taken in vacuum containers with EDTA as the anticoagulant. The blood was centrifuged at $1800 \mathrm{~g}$ for 10 min. After removal of the plasma, the erythrocytes were washed three times with $0.9 \% \mathrm{NaCl}$. Buffy coats 
were removed after each wash. Cell samples displaying hemolysis during washing or with a clot of extract residue during lipid extraction were excluded.

(2) Recommended procedure for lipid extraction. Erythrocytes were extracted with 12.5 volumes of methanol. Methanol was added drop-by-drop with stirring of the tube on a mixer until the extract residue turned brownishred. Thirty minutes later, 6.5 volumes of chloroform were added. The extraction was complete $1 \mathrm{~h}$ later. Occasional stirring of the extraction system was not necessary.

This procedure was used in the following experimental sections unless otherwise indicated. The addition of methanol before chloroform is expressed as methanol/ chloroform, while the addition of them as a mixture as methanol-chloroform. The nomenclature for individual gangliosides is in accordance with those used by Svennerholm. ${ }^{13}$

The addition of water to the methanol-chloroform mixture may cause the formation of a biphase. It is known that the distribution of lipids (cholesterol, phospholipids and gangliosides) is uneven in the upper waterrich and the lower chloroform-rich phases of a biphase system. The uneven lipid distribution leads to the incorrect estimation of lipid recovery when the lipid concentration in either phase is used as representative for the total solvent system. In this study, we wished to reveal the uneven distribution in an apparently monophase system. All experiments were therefore carried out in monophase systems and any samples showing a biphase were excluded.

(3) Ganglioside analysis. The intact extract supernatant (without filtration or centrifugation) was transferred to a tube, centrifuged at $1800 \mathrm{~g}$ for $10 \mathrm{~min}$, transferred to a second tube and evaporated to dryness under nitrogen. Non-ganglioside lipids and interfering materials (mainly pigment) in the crude lipid extract were removed by column chromatography, ${ }^{14}$ using 7.5 bed volumes of chloroform-methanol-water ( $65: 25: 4.5$ by vol). The ganglioside fraction was then eluted by using 10 bed volumes of chloroform-methanol-water $(30: 60: 20$ by vol). The samples were further purified by a modified resorcinol- $\mathrm{HCl}$ method $^{12}$ and sialic acids were quantified. A standard curve was established using $\mathrm{N}$-acetylneuraminic acid. Ganglioside content is expressed as nmol sialic acid.

Erythrocyte lipid extracts contain more pigment, sphingomyelin and sialic acid-poor gangliosides (GM series) but less of the GD and GT series, than other tissue extracts. We have disclosed that the pigment extraction increased with solvents containing more methanol and water, and with repeated extractions. For the ganglioside separation by thin-layer chromatography (TLC), both pigment and sphingomyelin had to be removed and for the total ganglioside analysis by our recommended procedure, ${ }^{12}$ the pigment has to be removed.

We observed that in the chromatographic column, the pigment and sphingomyelin were eluted together. To elute them, 7.5 volumes of chloroform-methanol-water $(65: 25: 4.5$ by vol) were needed. This volume was further adjusted with the pigment as a marker. The complete elimination of pigment and sphingomyelin and the retention of GM3 (the first ganglioside eluted) with this procedure was verified on TLC plates.

The recommended chloroform-silica gel purification ${ }^{12}$ was sufficient to remove interfering materials (including the pigment and sphingomyelin) for total sialic acid analysis. It was even used here in addition to the column chromatographic purification, since the extract purification with the column was not always sufficient.

(4) Individual gangliosides. After purification of the crude lipid extract by column chromatography, ${ }^{14}$ the ganglioside fraction was further purified by the partition-dialysis procedure of Svennerholm. ${ }^{15}$ The dialysate was evaporated and redissolved in $50 \mu \mathrm{l}$ of methanol-chloroform-water (30:60:4.5 by vol). Individual gangliosides were separated by a TLC procedure (an HPTLC Precoated Silica Gel 60 plate was developed in chloroformmethanol- $0.25 \% \mathrm{KCl} 50: 40: 10$ by vol). ${ }^{13}$ The gangliosides on the plates were identified, after staining with resorcinol- $\mathrm{HCl},{ }^{16}$ by comparing their migration rates with those of ganglioside standards. The relative distribution of the gangliosides was determined in duplicate plates with a densitometer (Onescanner-Ofoto ${ }^{\mathrm{TM}} 1.1$ ) coupled with Scan Analysis (68000) analytic programme on Macintosh IIsi computer. Tested with standard GM3, the coefficient of variation within the assays with the TLC procedure was $4.1 \%$ (in eight determinations), while the day-to-day variation was $5.4 \%$ over 4 days.

(5) Extra alcohol addition to reveal uneven distribution of gangliosides. The uneven distribution of gangliosides in the extraction system was tested by the addition of extra methanol to an 'extraction-finished' system. This method has been found useful in revealing the uneven distribution of cholesterol and phospholipids in polar solvent-nonpolar solvent-water mixtures. ${ }^{8}$

To monitor the uneven distribution of gangliosides in an extraction system, we assumed $R 1$ as the ganglioside recovery in the initial extraction and $R 2$ as that after an extra methanol addition. If the gangliosides had been completely extracted, only the ganglioside concentration in the extract supernatant should be diluted by the addition of the extra methanol; the total ganglioside recovery should not change, i.e., $R 1=R 2$. If $R 2>R 1$, it suggests a promoted extraction of gangliosides by the extra methanol, or an accumulation of gangliosides in the supernatant after the extra methanol addition. If $R 2<R 1$, on the other hand, it should be interpreted as an initial accumulation of gangliosides in the supernatant, unless there is evidence for ganglioside degradation after the extra methanol addition.

(6) Statistical analysis. In this study, the term recovery indicates the total amount of gangliosides extracted from erythrocytes, expressed as sialic acids $\mathrm{nM} / \mathrm{ml}$ packed erythrocytes. The packed cell volume was used as the reference for the sialic acid content since the coefficient of variation in the cell sampling (in a series of 10 determinations) was the lowest $(<3 \%)$. The coefficient of vari- 
ation in the cell number count and in the protein content determination was higher $(5-15 \%)$. In the one-step extraction, ganglioside recovery was calculated by multiplying the sialic acid concentration in the extract supernatant with the total solvent volume (including the original water volume in erythrocytes, e.g., $72 \%$ of wet weight). Values are expressed as mean \pm SD. The Student $t$-test was used for statistical analysis.

III. Ganglioside extraction by different methods. Erythrocytes from the same pool were extracted in quadruplicate for gangliosides by several available methods and by the recommended one-step procedure using 19 volumes of methanol/chloroform $(2: 1)$. The procedures in these different methods were followed exactly as in the reference (including the filtration or centrifugation after extraction). The Svennerholm ${ }^{13}$ method was understood to require solvent addition as a mixture.

The recommended method extracted the highest amount of gangliosides from human erythrocytes (Table 1). Generally speaking, a lower recovery was thought to be due to a loss of sialic acid-rich gangliosides (GD3 and GD1a). Applied on rat erythrocytes, total gan- glioside recovery by the Bligh and Dyer method and by the Svennerholm (1980) method was about $60 \%$ and $90 \%$, respectively, of that extracted by the recommended method.

The ganglioside extraction by the recommended method was judged complete when no more gangliosides were recovered by any of the following procedures: (1) with more than 50 volumes of the same solvent system extraction; (2) with the addition of an extra 11.25 volumes of methanol to the 'extraction-finished' extractions; (3) with repeated extractions (three times, 11.25 volumes of methanol-chloroform 2:1 each). Mechanical stirring of the extraction mixtures during the extraction or prolongation of the extraction up to $8 \mathrm{~h}$ did not significantly affect the ganglioside recovery. The ganglioside recovery was also regarded as reliable when the recovery was not influenced by an extra methanol addition, or by repeated extractions. With this method, the value for gangliosides from human erythrocytes was $28.5 \pm 9.7$ (sialic acid $\mathrm{nM} / \mathrm{ml}$ packed erythrocytes, $n=11$ ). Using human erythrocytes, the coefficient of variation within extractions was $3.9 \%$ (from the same pool in 12 extractions), while the day to day variation was $4.4 \%$ (blood was drawn from

Table 1. Erythrocyte ganglioside recovery extracted by different methods (expressed as sialtic acid $\mathrm{nM} / \mathrm{ml}$ packed erythrocytes).

\begin{tabular}{|c|c|c|c|c|c|c|}
\hline Extraction & Total sialic acid & GM 1 & GM3 & GD3 & GD 1a & Ref. \\
\hline Folch: $19 \vee M-C(1: 2)$ & $31.2(25)^{d}$ & 20.1 & 7.5 & 3.7 & & 1 \\
\hline Reed: $10 \vee M / C(1: 1)$, repeat three times & $32.8(40)^{c}$ & 22.4 & 8.4 & 4.3 & & 21 \\
\hline Portokalian: $20 \vee M / C(1: 1)$, then $10 \vee M-C(1: 1)$ & $36.7(18)^{c}$ & 22.1 & 8.9 & 4.6 & 0.8 & 22 \\
\hline $\begin{array}{l}\text { Bligh and Dyer: } 3 \vee M-C 52: 1), 1 \vee C, 1 \vee W^{a} \\
\text { Kates: } 1 \vee W, 7.5 \vee M-C(2: 1) \\
\text { then } 9.5 \vee M-C-W(2: 1: 0.8)\end{array}$ & $\begin{array}{l}20.4(19)^{e} \\
35.7(26)^{c}\end{array}$ & $\begin{array}{l}14.5 \\
21.2\end{array}$ & $\begin{array}{l}5.9 \\
8.2\end{array}$ & 4.3 & 0.6 & 23 \\
\hline $\begin{array}{l}\text { Svennerholm }(1980): 19.2 \vee \mathrm{W} / \mathrm{M} / \mathrm{C}(0.56: 2: 1) \text {, } \\
\text { then } 2 \vee \mathrm{W}, 8 \vee \mathrm{M}-\mathrm{C}(2: 1)\end{array}$ & $37.4(31)$ & 22.2 & 8.6 & 4.5 & 0.7 & 15 \\
\hline $\begin{array}{l}\text { Svennerholm (1989): } 3 \vee W, 20 \vee M-C-W(2: 1: 0.75) \text {, } \\
\text { repeated with } 20 \vee M-C-W(2: 1: 0.75)^{b}\end{array}$ & $25.6(13)^{e}$ & 18.1 & 6.3 & 2.0 & & 13 \\
\hline Rose and Oklander: $19 \vee \mathrm{W} / \mathrm{I} / \mathrm{C}(1: 11: 7)$ & $27.0(40)^{e}$ & 17.6 & 5.5 & 0.06 & & 6 \\
\hline Rokukawa: 18 v I-C (11:7) & $10.3(5)^{e}$ & 7.9 & 2.4 & & & 24 \\
\hline $\begin{array}{l}\text { Nudelman: } 8.5 \vee 1 / \text { Hexane } / W(5.5: 2: 1) \text {, } \\
\text { the } 5 \vee 1-\text { Hexane-W }(55: 20: 25)\end{array}$ & $33.2(35)^{c}$ & 20.6 & 8.0 & 4.0 & 0.6 & 25 \\
\hline Selvam: $18 \vee \mathrm{I}-$ Hexane $(2: 3)$ & $20.4(28)^{a}$ & 15.3 & 6.1 & 0.4 & & 26 \\
\hline Wesén: $20 \vee \mathrm{I}$ Cyclohexane $(1: 1)$ & $21.8(9)^{e}$ & 15.5 & 6.3 & 0.1 & & 27 \\
\hline Methanol alone (19 v) & $22.1(38)^{d}$ & & & & & \\
\hline Ethanol alone (19 v) & $15.2(9)^{a}$ & & & & & \\
\hline Isopropyl alcohol alone (19 v) & $7.7(9)^{a}$ & & & & & \\
\hline Recommended: $19 \vee \mathrm{M} / \mathrm{C}(2: 1)$ & $40.9(13)$ & 23.5 & 10.0 & 5.7 & 1.7 & \\
\hline
\end{tabular}

Erythrocytes from the same pool were extracted in quadriplicate. ${ }^{a}$ Biphase formed. Both phases were collected for ganglioside determination. ${ }^{b}$ Biphase formed. Upper phase was collected for sialic acid determination. Solvent/solvent: addition of alcohol before chloroform; solvent-solvent: addition of the solvent as a mixture. Abbreviations: $\mathrm{M}$, methanol; I, isopropyl alcohol; $\mathrm{C}$, chloroform; $W$, water and $v$, volume. ${ }^{c} p<0.05 .{ }^{d} p<0.01 .^{e} p<0.001$, when compared with the recommended one-step ( 19 volumes of methanol/chloroform $2: 1$ ) extraction. Values in parentheses are 10 times the standard deviation. The individual ganglioside composition was the mean of duplicate determinations. 
a given donor every day for 4 days, and erythrocytes extracted in triplicate).

$I V$. Procedures influencing ganglioside extraction and determination

(1) Solvent addition. The addition of methanol before chloroform to erythrocytes led to a relatively higher extraction of gangliosides than their addition as a mixture (Table 2). With the methanol added before chloroform, the maximum recovery was obtained with a methanol/ chloroform ratio of 1.5:1 and 2:1 (no significant difference between them).

(2) Total solvent-to-sample ratio. When erythrocytes from the same pool were extracted in triplicate with increasing volumes of methanol/chloroform (1:2 to $2: 1$ by vol.) (Fig. 1), small-volume solvents (solvent-to-sample ratio 7.5:1) gave a higher ganglioside recovery than largevolume solvents (solvent-to-sample ratio 11.25:1). The highest ganglioside recovery was, however, obtained with larger volumes of methanol/chloroform (2:1) (solvent-tosample ratio $\geq 15: 1$ ). Methanol alone failed to extract gangliosides completely.

In parallel experiments, 10 extra volumes of methanol were added to these 'extraction-finished' extractions. Such an addition reduced the initially higher recovery in the small-volume solvent, but not in the large-volume extractions of methanol/chloroform (2:1) solvents (data not shown). Therefore, we indicate the ganglioside extraction with the small-volume solvents as 'variable' and with the large-volume solvents as 'constant'. A constant extraction was reached with more than 45,40 , and 15 volumes of methanol/chloroform (1:2), (1:1) and (2:1) solvents, respectively (data not shown). No evidence was found for any ganglioside degradation after the extra methanol addition.

(3) Water addition. Ganglioside recovery was increased by the addition of water to the erythrocytes (Fig. 2). In methanol/chloroform (2:1), the maximal increase was by $18.9 \%(p<0.001$, compared with the constant recovery value) when the total water volume reached $24.4 \%$ (including the water in the samples). In methanol/chloro-

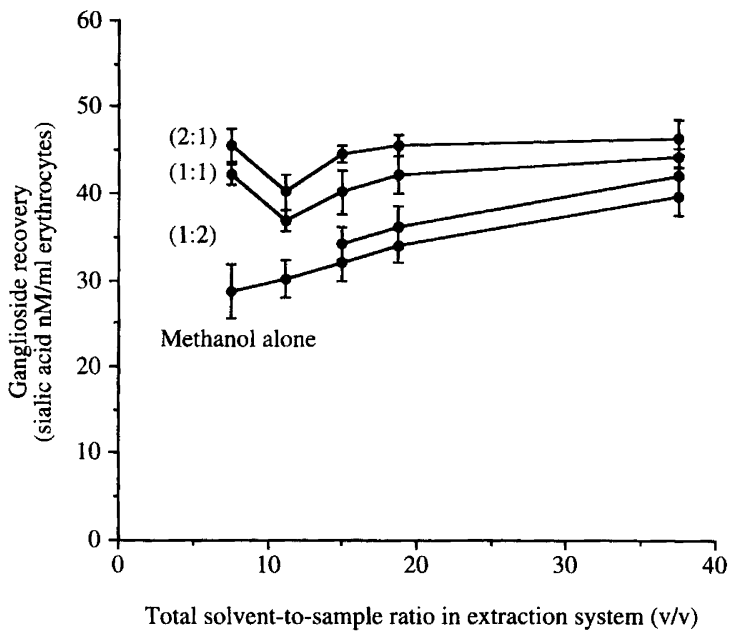

Fig. 1. Ganglioside extraction with increasing volumes of methanol or methanol/chloroform solvents. Aliquots of packed erythrocytes from the same pool were extracted in triplicate with increasing volumes of methanol alone or methanol/chloroform $(\mathrm{M} / \mathrm{C})(1: 2-2: 1)$. Methanol was added $30 \mathrm{~min}$ before chloroform. One hour after chloroform addition, gangliosides were analysed.

form (1:1), the maximal increase was $42.5 \%$ when water content was only $15.5 \%$. The addition of extra methanol (data not shown) to these 'extraction-finished' methanol/ chloroform extractions (e.g., alcohol/chloroform/alcohol extraction), however, reduced the ganglioside recovery by a maximum of $25.0 \%(p<0.001)$ and $35.7 \%(p<0.001)$, in methanol/chloroform $(2: 1)$ and (1:1), respectively.

(4) Ganglioside distribution after filtration and centrifugation. This was tested using rat brain gangliosides that had been extracted with 20 volumes of methanol/chloroform (2:1), purified by the column chromatography-partitiondialysis procedure (as described above), redissolved in methanol-chloroform $(2: 1)$, and stored at $-20^{\circ} \mathrm{C}$ before being used as a standard. The concentration of total lipidbound sialic acids in this standard was $121 \mathrm{nM} / 0.1 \mathrm{ml}$ solvent.

Table 2. Erythrocyte ganglioside recovery extracted by different methods of solvent addition (expressed as sialic acids $\mathrm{nM} / \mathrm{ml}$ packed erythrocytes).

\begin{tabular}{lll}
\hline & \multicolumn{2}{l}{ Addition of methanol and chloroform } \\
\cline { 2 - 3 } Extraction solvent & As a mixture & Methanol before chloroform \\
\hline Methanol : chloroform 1:2 & $16.4(22)$ & $23.7(10)^{a}$ \\
Methanol : chloroform 1:1 & $18.5(61)$ & $32.4(31)^{a}$ \\
Methanol : chloroform 1.5:1 & $21.0(20)$ & $34.1(29)^{b}$ \\
Methanol : chloroform 2: & $25.6(27)$ & $36.7(5)^{b}$ \\
Methanol : chloroform 3:1 & $31.0(11)$ & $33.6(34)$ \\
\hline
\end{tabular}

Aliquots of packed erythrocytes from the same pool were extracted in triplicate with 19 volumes of methanol/chloroform (1:2 to $3: 1$ ), with methanol added together with, or $30 \mathrm{~min}$ before chloroform. ${ }^{a} p<0.05 .{ }^{b} p<0.01$ compared with the values with solvent addition as a mixture, respectively. Values in parentheses are 10 times the standard deviation. 


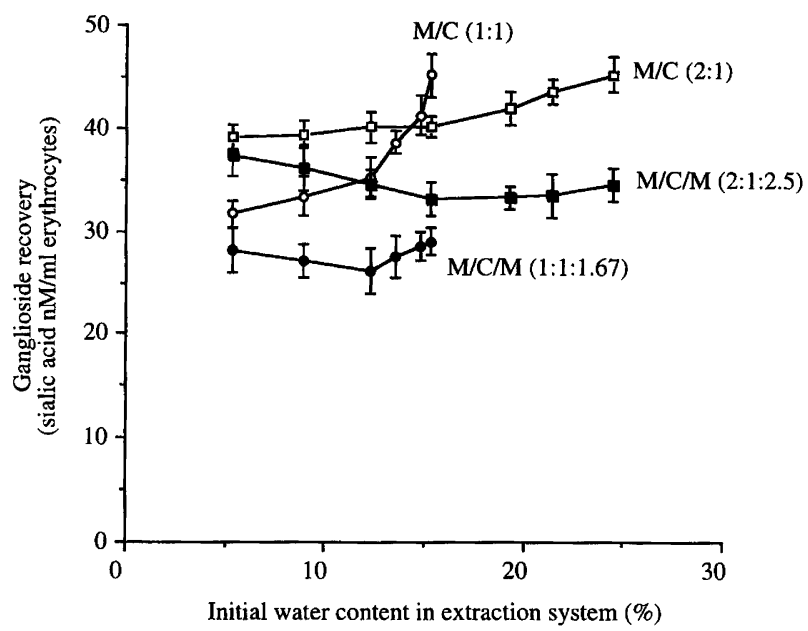

Fig. 2. Ganglioside extraction with increasing proportion of water. Two parallel experiments were performed. In the first, erythrocytes from the same pool were extracted in triplicate with 18 volumes of methanol/chloroform $(\mathrm{M} / \mathrm{C})(2: 1)$ or $(1: 1)$. Alcohol was added $30 \mathrm{~min}$ before chloroform. Before the extraction, water had been added to make the final water content in these extractions up to $24.4 \%$ or $15.5 \%$, respectively. In a parallel series, 12.5 volumes of extra methanol were added to these 'extraction-finished' systems (M/C/M extraction). The extraction lasted for $48 \mathrm{~h}$ and gangliosides in intact extract supernatants were analysed. The addition of extra methanol reduced the proportion of water in the 'extraction-finished' system but for the sake of comparison, the water proportion before the extra methanol addition (initial water content in the extraction) was still used.

(4a) After filtration. Gangliosides were lost in the glass wool after filtration of a methanol-chloroform solvent containing the prepared ganglioside standard (Fig. 3). The maximal decrease in ganglioside concentration in the filtrate was $10 \%, 30 \%$, and $44 \%(p<0.001$, versus control series) for methanol-chloroform (2:1), (1:1) and (1:2), respectively. This was found to be due to a true loss of gangliosides in the glass wool, rather than to a degradation of gangliosides in the solvents, since the gangliosides could be recovered from the pooled washings of the glass wool (data not shown). Generally speaking, the pooled washings contained relatively more sialic acid-rich gangliosides (GD and GT) (Table 3).

The ganglioside loss was dependent on the amount of glass wool used and the tightness of its packing. The relative composition of solvents was apparently changed after the filtration, since a biphase solvent mixture became a monophase. The addition of water to these solvent mixtures did not completely prevent the loss of gangliosides, since the concentration of gangliosides in the filtrate never reached the expected level (the concentration value in ganglioside solution prior to filtration) (Fig. 3).

To recover the lost gangliosides, thorough washing of the glass wool with methanol-chloroform-water $(2: 1: 0.75)$ after the filtration was needed.

(4b) After centrifugation. After centrifugation, the ganglioside concentration was increased in the upper fraction of methanol-chloroform (1:2 to 2:1) mixtures (Fig. 4).

Table 3. Individual gangliosides composition $(\%)$ in the filtrate and in pooled washings after filtration. ${ }^{a}$

\begin{tabular}{|c|c|c|c|c|c|}
\hline \multirow[b]{2}{*}{ Gangliosides } & \multirow[b]{2}{*}{ Control } & \multicolumn{2}{|l|}{$M-C(2: 1)$} & \multicolumn{2}{|l|}{ M-C (1:1) } \\
\hline & & Pooled washings & Filtrate & Pooled washings & Filtrate \\
\hline GM3 & 1.2 & & 0.9 & & 0.3 \\
\hline GM2 & 0.1 & & 0.9 & & 1.5 \\
\hline GM1 & 15.0 & 10.5 & 12.1 & 8.9 & 15.8 \\
\hline GD 1a & 41.7 & 43.0 & 34.9 & 42.2 & 33.0 \\
\hline GD 1b & 13.6 & 18.0 & 17.5 & 15.5 & 15.7 \\
\hline GT1b & 28.6 & 28.5 & 27.6 & 30.8 & 24.9 \\
\hline Others & & & 5.0 & 2.4 & 9.1 \\
\hline
\end{tabular}

${ }^{a}$ Purified rat brain gangliosides $(30 \mathrm{nM}$ sialic acids) were mixed with $4 \mathrm{ml}$ of methanol-chloroform either $(2: 1)$ or $(1: 1)$. The mixtures were filtered through glass wool that had been washed with $5 \mathrm{ml}$ of methanol-chloroform-water $(2: 1: 0.75)$ twice and dried before use. The filtrate and the pooled washings were evaporated separately. Individual gangliosides were separated by a TLC procedure. Duplicate separations were performed and mean values are given. Controls: ganglioside solution prior to filtration. 


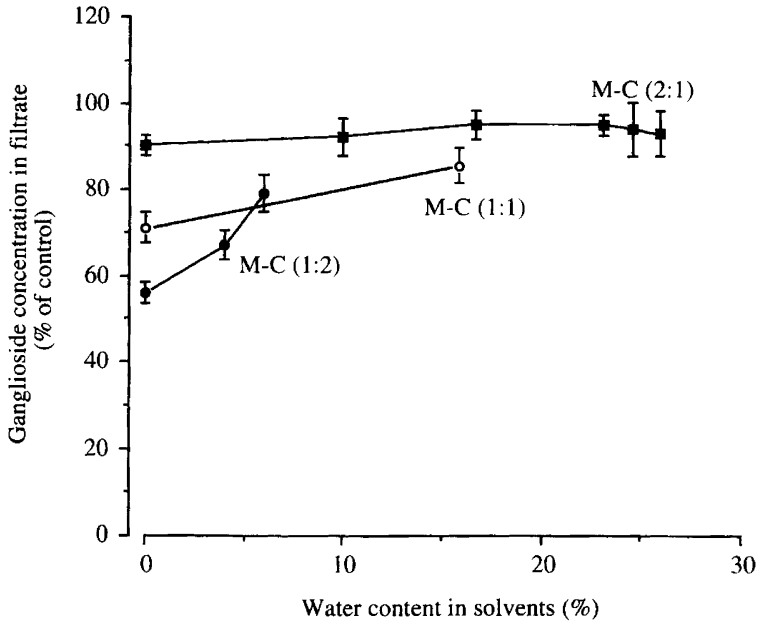

Fig. 3. Distribution of gangliosides in filtrate after filtration. Dried rat brain lipid extract was added with $4 \mathrm{ml}$ mixture of methanol-chloroform $(\mathrm{M}-\mathrm{C})(1: 2$ to $2: 1)$, then with increasing volumes of water. The mixtures were filtered through glass wool. Ganglioside concentrations were determined from the filtrate and values compared with those in the controls (the same ganglioside-containing solvents without filtration).

These fractions also contained relatively more sialic acidpoor gangliosides (GM) (data not shown). The uneven distribution phenomenon is apparently related to the water content in these solvents, and occurred even after overnight standing at room temperature.

The influence of the centrifugation on the ganglioside quantification in a sample with a small amount of residue was easy to prevent. It was sufficient to transfer the centrifuged solvent mixture to a different tube (centrifugedtransferred procedure). For a sample that contains a large amount of residue, we recommend that the initial extract supernatant (without filtration or centrifugation) be transferred to a different tube for centrifugation, and then that the centrifuged solvent be transferred to a second tube for quantification (transferred-centrifuged-transferred procedure). It should be reiterated that the ganglioside concentration obtained in this way reflects that only in the initial extract supernatant, but not necessarily that in the solvent remaining in the residue.

(5) Different alcohols. When the alcohol/chloroform ratio was kept at 1.5:1 and erythrocytes were extracted from the same pool in triplicate, methanol/chloroform extracted more gangliosides than both ethanol/chloroform and isopropyl alcohol/chloroform (Fig. 5), independent of the solvent-to-sample ratio.

(6) Repeated extractions. When erythrocytes from the same pool were extracted in quadruplicate by a one-step procedure using 18 volumes of either methanol/chloroform $(2: 1)$, or water/methanol/chloroform $(0.75: 2: 1)$, the ganglioside recovery was $34.7 \pm 1.2$ and $39.1 \pm 1.1 \mathrm{nM} / \mathrm{ml}$ packed erythrocytes, respectively. The recovery was $32.2 \pm 1.5$ for double methanol/chloroform $(2: 1)$ extrac-

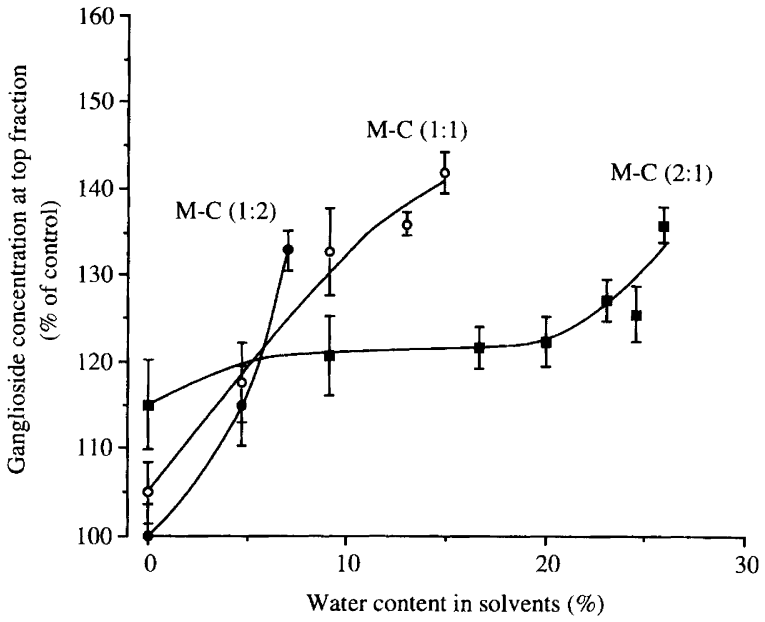

Fig. 4. Distribution of gangliosides in the solvent top fraction after centrifugation. Dried rat brain lipid extract was added in triplicate with $4 \mathrm{ml}$ methanol-chloroform (M-C) mixture (1:2$2: 1$, then with increasing volumes of water. The mixtures were centrifuged at $1800 \mathrm{~g}$ for $20 \mathrm{~min}$. Top fractions $(0.2 \mathrm{ml})$ were taken within $30 \mathrm{~min}$ for ganglioside analysis. The results were compared with those in a control series (the same ganglioside-containing solvents without centrifugation).

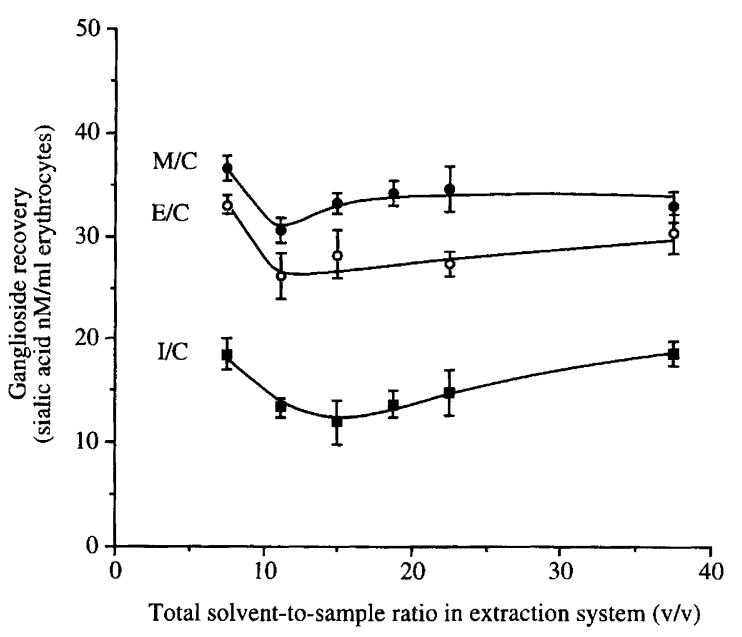

Fig. 5. Ganglioside extraction with different alcohol/chloroform systems. Aliquots of packed erythrocytes from the same pool were extracted in triplicate with increasing volumes of methanol/chloroform (M/C 1.5:1), ethanol/chloroform (E/C 1.5:1) or isopropyl alcohol/chloroform (I/C 1.5:1). Alcohols were added $30 \mathrm{~min}$ before chloroform. Gangliosides were analysed $1 \mathrm{~h}$ after chloroform addition.

tions (18 volumes each), and $28.8 \pm 2.1 \mathrm{nM} / \mathrm{ml}$ packed erythrocytes, for double water/methanol/chloroform $(0.75: 2: 1)$ extractions (22.5 volumes each). In these repeated extractions, extract supernatants were collected after centrifugation at $1800 \mathrm{~g}$ for $10 \mathrm{~min}$. The extracts were pooled and evaporated before ganglioside analysis. 


\section{Discussion}

In agreement with our previous findings on the extraction of cholesterol and phospholipids from erythrocytes, ${ }^{8,9}$ data in this study show that there is also an uneven distribution of gangliosides in an apparently monophasic methanol-chloroform-water extraction system. The small-volume solvent ( 7.5 volumes) extraction showed higher ganglioside recovery than the large-volume solvent (11.25 volumes) extraction (Fig. 1). The addition of water to the methanol/chloroform extraction system (Fig. 2) revealed an increased ganglioside recovery in relation to the relative water content in the extraction system. However, the addition of methanol to the 'extraction-finished' extraction systems reduced the ganglioside recovery. The initial gangliosides recovery $(R 1)$ was lower than the recovery value $(R 2)$ after the extra methanol addition.

Most likely, the uneven distribution of solvents in the extraction system is due to their different gravity (chloroform $>$ water $>$ methanol). Gangliosides in the upper water-rich solvents tend to aggregate and float, and thereby result in their higher concentration in the upper solvent. A portion of water may also bind to the residue surface, as evidenced by the residue particles floating in the extraction system. Such binding prevents full contact by the solvents for a complete extraction. The extra methanol addition to the extraction systems eliminated the uneven distribution of solvent, ${ }^{9}$ reduced the ganglioside concentration in the upper solvent, but failed to promote the ganglioside extraction by this binding of the water in the residue surface.

We have previously reported that a filtration of lipidcontaining solvents through glass wool results in an increased cholesterol concentration in the filtrate. ${ }^{9}$ In this study, however, the ganglioside concentration was reduced after such a filtration (Fig. 3). This could hardly be due to a degradation of gangliosides, but rather to their loss, since gangliosides were recovered in the glass wool and we revealed no evidence for a ganglioside degradation. Gangliosides and water may compete for binding sites on the glass wool. Furthermore, the addition of water to the solvent mixture appeared to prevent this ganglioside loss.

Centrifugation of a methanol-chloroform-water mixture makes the upper solvent richer in water and the bottom solvent enriched in chloroform. Owing to the high affinity of the polar gangliosides to water, gangliosides may tend to dissolve or aggregate in the upper water-rich solvent to give a higher concentration there than in the lower portion (Fig. 4).

The influence of solvent addition (as a mixture or separately with alcohol first) on the ganglioside extraction was similar to that observed in the experiments on cholesterol extraction. ${ }^{8}$ Thus, our data are in agreement with those earlier findings that state that polar lipids are more difficult to extract. ${ }^{17-20}$ Our data also show that a polar alcohol (methanol) is required in an alcohol-chloroform system for complete extraction of gangliosides as well. ${ }^{15}$
When the above-mentioned weaknesses in the extraction procedures were controlled, the recommended onestep extraction procedure using more than 19 volumes of methanol/chloroform (2:1) appeared to be a simple procedure with the best recovery of all lipids when compared with a number of other methods (Table 1, Fig. 1).

We conclude then that the uneven distribution of solvent and the extracted lipids in a methanol-chloroformwater system is a common phenomenon in the extraction of not only neutral (cholesterol) but also polar lipids (gangliosides). The extraction and quantification of these lipids is furthermore negatively influenced by the addition of solvents as a previously prepared mixture and by the use of less polar alcohols such as isopropyl alcohol. These factors are uncontrolled in earlier suggested procedures and are most likely the cause of the large variation in lipid extractions that are experienced when lipids are either extracted from a given tissue by different methods or when variable tissues are extracted by one given method.

\section{References}

1. Folch, J., Lees, M. and Sloane-Stanley, G. H. J. Biol. Chem. 226 (1956) 497.

2. Bligh, E. G. and Dyer, W. J. Can. J. Biochem. Physiol. 37 (1959) 911.

3. Erickson, B. N., Williams, H. H., Hummel, F. C. and Macy, I. G. J. Biol. Chem. 118 (1937) 15.

4. Farquhar, J. W. Biochim. Biophys. Acta 60 (1962) 80.

5. Westerman, M. P., Pierce, L. E. and Jensen, W. N. J. Lab. Clin. Med. 62 (1963) 394.

6. Rose, H. G. and Oklander, M. J. Lipid Res. 6 (1965) 428.

7. Nelson, G. J. In: Perkins, E. G., Ed., Analysis of Lipids and Lipoproteins, American Oil Chemist's Society, Champaign Illinois, 1975, pp. 1-22.

8. Wang, W. Q. and Gustafson, A. Acta Chem. Scand. 48 (1994) 753.

9. Wang, W. Q. and Gustafson, A. J. Lipid Res. 35 (1994) 2143.

10. Hakomori, S.-I. In: Kanfer, J. N. and Hakomori, S.-I., Eds., Handbook of Lipid Research. Sphingolipid Biochemistry, Plenum Press, New York and London, 1983.

11. Mukhin, D. N., Prokazova, N. V., Bergelson, L. D. and Orekhov, A. N. Atherosclerosis 78 (1989) 39.

12. Wang, W. Q. and Gustafson, A. Acta Chem. Scand. 49 (1995) 138.

13. Svennerholm, L., Boström, K., Fredman, P., Månsson, J.E., Rosengren, B. and Rynmark, B.-M. Biochim. Biophys. Acta 1005 (1989) 109.

14. Davidsson, P., Fredman, P., Collins, V. P., von Holst, H., Månsson, J.-E. and Svennerholm, L. J. Neurochem. 53 (1989) 705.

15. Svennerholm, L. and Fredman, P. Biochim. Biophys. Acta 617 (1980) 97.

16. Svennerholm, L. Biochim. Biophys. Acta 24 (1957) 604.

17. Saito, M., Tanaka, Y. and Ando, S. Anal. Biochem. 132 (1983) 376.

18. Freyburger, G., Heape, A., Gin, H., Biosseau, M. and Cassagne, C. Anal. Biochem. 171 (1988) 213.

19. Eder, K., Reichlmayr-Lais, A. M. and Kirchgebner, M. Clin. Chim. Acta 219 (1993) 93.

20. Naito, H. K. and David, J. A. In: Story J. A., Ed., Lipid Research Methodology, Liss, 1984. pp. 1-76.

21. Reed, C. F., Swicher, S. N., Marinetti, G. V. and Eden, E. G. J. Lab. Clin. Med. 56 (1960) 281. 
22. Portoukalian, J., Zwingelstein, G., Abdul-Malak, N. andDoré, J. F. Biochem. Biophys. Res. Commun. 85 (1978) 916.

23. Kates, M. Techniques of Lipidology: Isolation, Analysis and Identification of Lipids, 2nd revised ed., Elsevier, Amsterdam, New York, Oxford 1986, pp. 100-114.

24. Rokukawa, C., Kushi, Y., Ueno, K. and Handa, S. J. Biochem. 92 (1982) 1481.
25. Hakomori, S.-I. In: Kanfer, J. N. and Hakomori, S.-I., Eds., Handbook of Lipid Research. Sphingolipid Biochemistry, Plenum Press, New York and London 1983, pp. 1-165.

26. Selvam, R. and Radin, N. Anal. Biochem. 112 (1981) 338.

27. Wesén, C. and Mu, H. J. Chromatogr. 625 (1992) 257.

Received April 13, 1995. 